\title{
Chronische Nierenerkrankungen
}

C hronische Nierenerkrankungen sind vergleichsweise selten. In den letzten Jahren stiegen ihre Inzidenz und Prävalenz vor allem infolge zunehmender Atherosklerose und Diabetes mellitus Typ 2 an. Auch immunologische Systemerkrankungen mit Nierenbeteiligung wie unterschiedliche Vaskulitiden und systemischer Lupus erythematodes spielen eine Rolle.

Das vorliegende Heft des klinikarzt soll an den Beispielen der Nierenarterienstenose (NAST) und der renovaskulären Hypertonie (RVH), der Lupusnephritis und der Bedeutung der Hypertonie für die Progression von unterschiedlichen Nierenerkrankungen zeigen, welche Präventions- und Therapiemöglichkeiten in den letzten Jahren erarbeitet wurden. Dabei gehen die Autoren nicht nur auf die Pathophysiologie und die Diagnostik der Nierenarterienstenose und der renovaskulären Hypertonie ein, sondern auch auf aktuelle Therapiekonzepte.

Neben der seit vielen Jahren akzeptierten Cyclophosphamidtherapie bei der proliferativen Lupusnephritis zur Prävention einer dialysepflichtigen Niereninsuffizienz können Sie sich über zum Teil etablierte, zum Teil zukunftsweisende Therapieverfahren informieren. Noch immer besteht bei der Lupusnephritis eine große Diskrepanz zwischen der Häufigkeit der Erkrankung und den nur wenigen Studien als Grundlage für eine evidenzbasierte Therapie.

Das in den letzten Jahren vielfach diskutierte Thema der Beeinflussung der Progression von Nierenerkrankungen durch eine optimale Hypertoniebehandlung mit Zielblutdruckwerten von maximal 130/80 mmHg fasst ein weiterer Beitrag übersichtlich zusammen. Neben den neuesten Studien bei Typ-2-Diabetikern wird auch auf nichtdiabetische chronische Nierenerkrankungen eingegangen.

Zur Nierenersatztherapie - die in Deutschland an mehr als 50000 Patienten durchgeführt wird - werden zwei weniger populäre Verfahren beschrieben. Die automatisierte Peritonealdialyse (APD) ermöglicht eine sehr effektive langjährige Nierenersatztherapie, die mit zahlreichen Vorteilen für den Patienten verbunden ist. Trotzdem wird dieses Verfahren zur Heimdialysetherapie in Deutschland noch zu wenig eingesetzt. Es wird eine aktuelle Information sowohl über die modernen apparativen Möglichkeiten als auch über die Vorteile in Bezug auf eine adäquate Dialyse gegenüber der chronisch ambulanten Peritonealdialyse (CAPD) gegeben.

An Bedeutung gewonnen hat auch die Nierentransplantation von verwandten und nichtverwandten Lebendnierenspendern. Dabei spielen das 1997 in Kraft getretene Transplantationsgesetz ebenso wie die unverändert geringe Verfügbarkeit von Spenderorganen Verstorbener in Deutschland eine Rolle. Im Jahre 2002 war der Anteil der Lebendspender in Deutschland bei gleichbleibender Gesamtzahl fast 20\%. Die Autoren präsentieren die Ergebnisse ebenso wie die Erfahrungen mit der präemptiven (vor Einleitung einer Dialyse) durchgeführten Lebendspendetransplantation, nicht ohne auf die unbedingt notwendige Steigerung der postmortalen Organspende hinzuweisen.

Alle Themen werden vor dem Hintergrund langjähriger klinischer Erfahrungen an einem großen Krankengut der Düsseldorfer Klinik dargestellt.

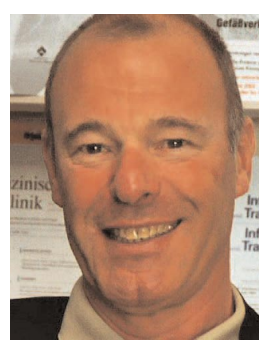

Prof. Dr. B. Grabensee, Düsseldorf

Gasteditor)

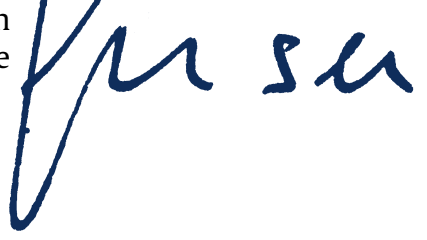

